\section{Mortalidade de homens jovens por agressões no Brasil, 2010-2014: estudo ecológico}

\author{
Mortality from assault in young men in Brazil, \\ 2010-2014: an ecological study
}

\section{Mortalidad de hombres jóvenes por agresiones en Brasil, 2010-2014: estudio ecológico}

Alice Cristina Medeiros Melo 1

Gabriela Drummond Marques da Silva 1

Leila Posenato Garcia 1,2

doi: 10.1590/0102-311X00168316

\section{Resumo}

O objetivo foi analisar a associação entre o risco de morte por agressões em jovens do sexo masculino e características sociodemográficas dos municípios brasileiros. Estudo ecológico tendo como unidades de análise os $1.651 \mathrm{mu}$ nicípios com mais de 20.000 habitantes. Foram utilizados dados do Sistema de Informação sobre Mortalidade (SIM) e indicadores obtidos do Censo Demográfico 2010 e do Atlas do Desenvolvimento Humano no Brasil. Razões de taxas de mortalidade foram estimadas por modelo de regressão binomial negativa. No período de 2010-2014, foram registrados 127.137 óbitos por agressão a jovens de 15-29 anos de idade do sexo masculino. A taxa corrigida de mortalidade foi 133,3/100 mil habitantes no conjunto dos municípios (mediana 71,5/100 mil habitantes). A taxa foi maior à medida que aumentou o porte populacional dos municípios. Razões de taxas mais elevadas no modelo ajustado foram observadas nos municipios mais urbanizados (1,95; IC95\%: 1,70-2,23), em categorias intermediárias de desigualdade de renda (1,10; IC95\%: 1,01-1,20) e proporção de pobreza (1,69; IC95\%: 1,511,89), com menor proporção de jovens frequentando o Ensino Médio (2,05; IC95\% 1,83-2,30), maior proporção de jovens de 18-24 anos desocupados (1,27; IC95\%: 1,16-1,40) e maior número de mulheres em relação ao de homens (1,28; IC95\% 1,05-1,58). A mortalidade de jovens do sexo masculino por agressão foi elevada, especialmente nos municípios maiores, mais urbanizados e com maior proporção de jovens buscando emprego e fora do ensino médio. Evidencia-se a relevância das politicas sociais para o enfrentamento da violência entre jovens.

Homicídio; Violência; Desigualdades em Saúde; Homens
Correspondência

A. C. M. Melo

Programa de Pós-graduação em Saúde Coletiva, Universidade de Brasília.

Campus Universitário Darcy Ribeiro s/n, Brasília, DF 70910-900, Brasil.

acnmedeiros@gmail.com

1 Programa de Pós-graduação em Saúde Coletiva, Universidade de Brasília, Brasília, Brasil.

2 Instituto de Pesquisa Econômica Aplicada, Brasília, Brasil. 


\section{Introdução}

Mudanças sociais, econômicas e políticas globais ocorridas nas últimas décadas afetaram os padrões de saúde da juventude no mundo. Entre jovens, as causas externas são as principais causas de morte e importantes causas de lesões, sequelas e incapacidades 1,2. Segundo o Observatório Regional de Saúde da Organização Pan-Americana da Saúde (OPAS), as agressões foram a principal causa de morte de jovens entre 15 e 29 anos de idade na região das Américas, em 20123.

No Brasil, no período de 2000-2012, essas também foram a principal causa dos óbitos entre jovens 4 . A elevada mortalidade relacionada à violência é atribuída aos homicídios em contextos urbanos no país, cujos perpetradores e vítimas são principalmente os homens jovens, tendo nas desigualdades sociais um de seus principais determinantes 5 . Essa realidade difere de grande parte dos países membros da Organização Mundial da Saúde (OMS), onde os óbitos por agressões estão relacionados a conflitos civis 6.

A literatura evidencia a predominância do sexo masculino tanto como vítima quanto como autor de agressões 1,7,8. Em um estudo sobre mortes por agressões na região das Américas, a sobremortalidade masculina foi observada em todos os países, no período de 1999-2009 1. O mesmo foi observado em jovens no Brasil, onde para cada mulher, aproximadamente 12 homens morreram por agressões no período de 1996-2007 9 .

Um trabalho sobre a morbimortalidade de jovens por agressões no Brasil, no período de 19962007, evidenciou uma razão de sexos masculino/feminino de 11,6 no número de óbitos, 4,5 no de internações e 2,8 no número de atendimentos de emergência ${ }^{9}$. Ademais, um estudo realizado com dados das vítimas de violência, com idades entre 15 e 29 anos, atendidas em serviços de urgência e emergência do Brasil, no ano de 2011, apontou maior proporção de lesões mais graves e óbitos nas primeiras 24 horas entre os homens em comparação às mulheres 10.

No Brasil, não obstante as tendências estacionárias das taxas de mortalidade geral de jovens no período de 2000-2012, permanecem importantes diferenças regionais. Houve tendência crescente entre os homens nas regiões Nordeste e Sul, e decrescente, em ambos os sexos, na Região Sudeste 4. Diversos estudos têm apontado hipóteses concorrentes para explicar as tendências na mortalidade por agressões, que tangenciam, além dos fatores individuais, características dos municípios, como desemprego, analfabetismo, pobreza e desigualdades sociais, que contribuem diretamente para o aumento da violência 11,12,13.

No Brasil, um trabalho sobre características dos municípios e o risco de homicídios em homens adultos de 20-39 anos de idade, no período de 2007-2010, revelou riscos superiores nos municípios com maior porte populacional, maior urbanização, maiores taxas de fecundidade, baixa proporção de alfabetizados, maior desigualdade econômica, e naqueles com categorias intermediárias das taxas de pobreza e de renda média per capita 14.

Tendo em vista a hipótese de que as características dos municípios estão associadas à ocorrência mortes de homens jovens por agressão, e a recente aprovação do Estatuto da Juventude no Brasil - que inclui entre seus princípios e diretrizes a promoção da vida segura e da cultura da paz, bem como a necessidade de gestão da informação e produção de conhecimento sobre juventude 15, justifica-se a realização de estudo ecológico sobre o tema.

Este trabalho tem como objetivo analisar a associação entre o risco de morte por agressões em jovens do sexo masculino, no período de 2010-2014, e características sociodemográficas dos municípios brasileiros.

\section{Método}

\section{Desenho do estudo}

Trata-se de um estudo ecológico transversal, cujas unidades de análise foram os 1.651 municípios brasileiros, com mais de 20 mil habitantes. 


\section{Fontes de dados}

Foram utilizados dados do Sistema de Informação sobre Mortalidade (SIM), do Censo Demográfico 2010, além do Atlas do Desenvolvimento Humano no Brasil (dados disponibilizados pelo Programa das Nações Unidas para o Desenvolvimento - PNUD).

\section{Desfecho}

A variável dependente do estudo é a taxa de mortalidade corrigida de homens jovens (15-29 anos) por agressões nos municípios, no período de 2010-2014.

Considerando-se a subestimação decorrente da má classificação da causa da morte no SIM e do sub-registro de óbitos neste sistema, sobretudo no que se refere às mortes violentas, optou-se por calcular taxas de mortalidade corrigidas. Essa correção foi realizada em duas etapas. A primeira, para a redistribuição dos óbitos por causas externas cuja intenção é indeterminada, foi realizada de acordo com a proposta de Borges \& Cano ${ }^{16}$, empregada previamente no cálculo do Índice de Homicídios na Adolescência. Essa proposta é baseada em trabalho prévio ${ }^{17}$, que revelou que praticamente todos os óbitos por arma de fogo e/ou instrumentos perfurocortantes que foram classificados como de intenção indeterminada podem ser considerados como intencionais (homicídio ou suicídio) e que aproximadamente $10 \%$ dos óbitos com intenção indeterminada por outros meios podem ser classificados como homicídios.

Na primeira etapa, inicialmente, foram somados todos os óbitos classificados nos seguintes códigos da Classificação Internacional de Doenças - 10a Revisão (CID10): agressões/homicídios (A = X85 a Y09 e Y871) e lesões resultantes de intervenções legais e operações de guerra ( $\mathrm{B}=$ Y350 a Y357). Os óbitos por lesões autoprovocadas intencionalmente (C = X60 a X84 e Y870) foram considerados exclusivamente para o cálculo da proporção dos óbitos por agressão em relação ao total dos óbitos por agressão mais aqueles por lesões autoprovocadas intencionalmente $(\mathrm{P}=\mathrm{A} /[\mathrm{A}+\mathrm{C}])$. Essa proporção foi multiplicada ao número de óbitos com intenção indeterminada por arma de fogo (Y22 a Y24) e arma branca (Y28), para a obtenção dos valores D e E. Considerou-se, ainda, 10\% do total de óbitos com intenção indeterminada por outros meios (Y10 a Y21, Y25 a Y27, Y29 a Y34, Y872), equivalente ao valor $\mathrm{F}$. O número total de óbitos resultante da primeira etapa de correção foi obtido por meio da soma dos valores A, B, D, E e F (Figura 1). A segunda etapa de correção consistiu da aplicação dos fatores de correção das taxas de mortalidade produzidos por Szwarcwald et al. 18 para a correção de sub-registro, uma vez que a cobertura do SIM não atinge 100\% e não é homogênea entre as regiões brasileiras 19. Foram empregados os fatores de correção médios por Unidade da Federação (UF), derivados de um estudo realizado em 2008, que estimou as coberturas dos sistemas de informações vitais com base em busca ativa de óbitos em uma amostra de 129 municípios localizados na Região Nordeste e na Amazônia Legal 18.

\section{Cálculo das taxas de mortalidade}

A taxa de mortalidade corrigida de homens jovens por agressões em cada município brasileiro foi calculada dividindo-se o número total de óbitos corrigido na população de referência, segundo local de residência no quinquênio 2010-2014, pela somatória da população de referência no mesmo período, multiplicando-se por 100 mil. Os denominadores (população de homens de 15-29 anos de cada município no período 2010-2014) foram obtidos multiplicando-se o tamanho da população total residente em cada ano - estimado pelo Instituto Brasileiro de Geografia e Estatística (IBGE) para o Tribunal de Contas da União (TCU) - pela proporção de jovens do sexo masculino na população total, segundo dados do Censo Demográfico 2010. Essa abordagem foi adotada devido à indisponibilidade de dados sobre o tamanho da população dos municípios brasileiros segundo sexo e faixa etária, nos anos posteriores a 2010, até o momento da realização deste estudo.

Foram calculadas as taxas agregadas em quinquênio buscando alcançar maior estabilidade dos indicadores calculados e dos riscos estimados. Análises preliminares subsidiaram a decisão quanto à exclusão dos municípios com até 20 mil habitantes (3.914 municípios), devido ao pequeno número de óbitos por agressão registrado no SIM e possíveis diferenças no perfil da mortalidade, que aumenta- 
Figura 1

Procedimento adotado na primeira etapa de correção das taxas de mortalidade de jovens por agressões.

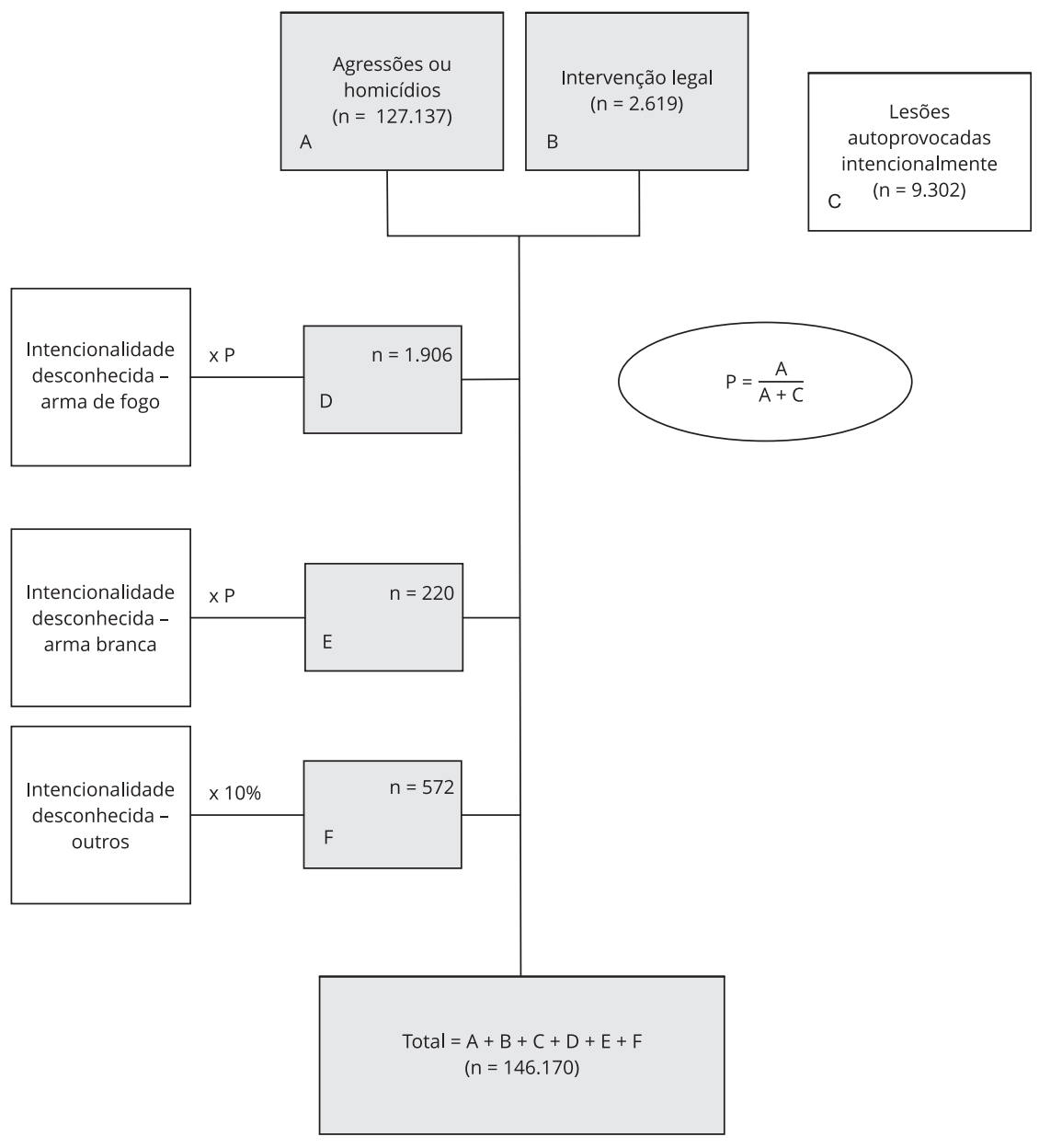

Notas: o valor C (número de óbitos por lesões autoprovocadas intencionalmente) foi considerado exclusivamente para o cálculo da proporção dos óbitos por agressão em relação ao total dos óbitos por agressão mais aqueles por lesões autoprovocadas intencionalmente $(P=A /[A+C])$. $O$ total refere-se ao número de óbitos após a primeira etapa de correção, para considerar a redistribuição dos óbitos por causas externas cuja intenção é indeterminada.

riam a probabilidade de detectar associações não verdadeiras. Em 2010, os municípios com mais de 20 mil habitantes concentravam 83,4\% da população jovem brasileira e 92,9\% das mortes de jovens do sexo masculino por agressões, embora correspondessem a 29,7\% do total de municípios do Brasil (tabulação das autoras, baseando-se em dados do SIM).

\section{Variáveis independentes}

As variáveis independentes, que caracterizam os municípios brasileiros estudados, foram: porte populacional (em 2010); proporção da população residente em área urbana (\% da população residente por situação do domicílio "urbano" segundo os municípios, em 2010); proporção de população de baixa renda (\% de pessoas com renda domiciliar per capita igual ou inferior a R\$ 140,00 mensais, equivalente a $1 / 2$ salário mínimo vigente em agosto de 2010); renda 20/40 (razão entre a renda dos 20\% 
mais ricos e os $40 \%$ mais pobres - é uma medida do grau de desigualdade existente na distribuição de indivíduos segundo a renda domiciliar per capita, em 2010); proporção de frequência líquida ao Ensino Médio (\% de jovens na faixa etária de 15-17 anos frequentando o ensino médio regular); proporção de desocupação na faixa etária de 18-24 anos (\% da população economicamente ativa na faixa etária que não estava ocupada na semana anterior à data do Censo 2010, mas havia procurado trabalho ao longo do mês anterior à data desta pesquisa); razão de sexo (razão entre o número de homens e o número de mulheres na população, em 2010). Todas as variáveis que caracterizam os municípios brasileiros foram originadas das bases de dados do Censo Demográfico 2010 e do Atlas do Desenvolvimento Humano no Brasil.

Para a análise, as variáveis foram categorizadas com base em tercis aproximados: proporção da população residente em área urbana: $\leq 65 \% ; 65 \%-90 \% ; \geq 90 \%$; proporção da população de baixa renda: $\leq 7 \% ; 7 \%-30 \% ; \geq 30 \%$; renda 20/40: $\leq 9 ; 9-12 ; \geq 12$; taxa de frequência líquida ao ensino médio - 15-17 anos: $\geq 35 \% ; 35 \%-45 \% ; \leq 35 \%$; taxa de desocupação - $18-24$ anos: $\leq 12 \%$; $12 \%-16 \% ; \geq 16 \%$.

Foram exceções "porte populacional" em que utilizou-se a mesma categorização adotada pelo IBGE (pequeno porte 2: 20.001-50.000 habitantes; médio porte: 50.001-100.000 habitantes; grande porte: 100.001-900.000 habitantes; metrópoles: mais de 900.000 habitantes) e "razão de sexo", em que foi utilizada a categorização: $>1,1=$ maior número de homens do que de mulheres, 1-1,1 = número de homens e mulheres aproximadamente igual $\mathrm{e}<1=$ número de mulheres maior do que o de homens.

\section{Análise dos dados}

Modelos de regressão binomial negativa brutos e ajustados foram construídos para estimar razões de taxas de mortalidade (RTM) e seus respectivos intervalos de 95\% de confiança (IC95\%). A opção pelo modelo de regressão binomial negativa justifica-se em função das propriedades da variável dependente, que apresentou grande dispersão, o que impossibilitou o uso da Regressão de Poisson, inicialmente testada. A distribuição binomial negativa é indicada nos casos de excesso de dispersão dos dados, ou seja, quando a variância é maior do que a resposta média 20.

Para a construção do modelo ajustado, considerou-se a significância estatística das variáveis independentes na análise bruta $(\mathrm{p}<0,05)$ e a ausência de colinearidade entre as variáveis (verificada pelo valor de inflação da variância - VIF $<5$ e com base em pressupostos teóricos) 21 . Foram formados grupos de variáveis independentes não colineares e construídos múltiplos modelos de regressão contendo as variáveis de cada grupo. Foi escolhido como modelo final aquele que apresentou o menor AIC (critério de informação de Akaike) 20.

A variável "porte populacional" ocultou a relação entre as variáveis que mensuram a desigualdade e o desfecho, levando a valores de p não significativos. Considerando-se a importância de avaliar a associação entre esses fatores e a taxa de mortalidade por agressão nos jovens, optou-se por não incluir "porte populacional" no modelo final. Foi construído um boxplot para ilustrar a dispersão das taxas de mortalidade segundo o porte populacional dos municípios. Durante os exercícios de modelagem, verificou-se não ser possível incluir a variável região no modelo final por causa da heterogeneidade nos valores da variável dependente entre as UF, mesmo entre aquelas que pertencem à mesma região. A variável UF foi testada, todavia, não foi obtido ajuste adequado do modelo, de modo que esta também não fez parte do modelo final.

Para avaliar a qualidade do ajuste do modelo, foram analisados os gráficos normais de probabilidades para os resíduos deviance referentes ao modelo log-linear binomial negativo. Além disso, foram plotados gráficos dos resíduos deviance versus o logaritmo dos valores ajustados e boxplot dos resíduos em relação às categorias das variáveis explicativas. Por fim, foi verificada a presença de pontos influentes e de alavanca, por meio da distância de Cook e dos elementos da diagonal principal da matriz de projeção $\mathrm{H}$ (matriz hat) 20.

\section{Softwares utilizados}

A tabulação dos dados foi feita com o programa Excel 2010 (Microsoft Corp., Estados Unidos). Para a análise dos dados utilizou-se o aplicativo Stata versão 12 (StataCorp LP, College Station, Estados Unidos). 


\section{Considerações éticas}

O presente trabalho utilizou exclusivamente dados secundários de agregados obtidos de fontes de acesso público. A proposta deste estudo foi dispensada de apreciação por Comitê de Ética em Pesquisa, em conformidade com os princípios da ética em pesquisa envolvendo seres humanos, constantes na Resolução do Conselho Nacional de Saúde (CNS) no 510, de 7 de abril de 2016.

\section{Resultados}

No período de 2010-2014, nos 1.651 municípios brasileiros com mais de 20 mil habitantes, foram registrados 127.137 óbitos por agressão a jovens de 15-29 anos de idade do sexo masculino no SIM. Após correção, o número de óbitos elevou para 146.170 e a taxa de mortalidade para o conjunto de municípios foi de 133,3. As taxas de mortalidade por 100 mil habitantes não corrigidas variaram entre 42,9 (São Paulo) e 312,1 (Alagoas), e as taxas corrigidas variaram entre 49,0 e 340,2, nas mesmas UF. O percentual de correção foi 15\% para o Brasil, variando entre 0,4\% no Distrito Federal e 32,3\% no Maranhão (Tabela 1).

Tabela 1

Número de óbitos (bruto e corrigido) por agressão e taxa de mortalidade (bruta e corrigida) por agressão de jovens de 15-29 anos de idade (por 100 mil habitantes), segundo Unidades da Federação. Municípios brasileiros com mais de 20 mil habitantes, 2010-2014.

\begin{tabular}{|c|c|c|c|c|c|c|}
\hline Unidades da Federação & $\begin{array}{l}\text { Número de } \\
\text { municípios }\end{array}$ & $\begin{array}{l}\text { Número de } \\
\text { óbitos não } \\
\text { corrigido }\end{array}$ & $\begin{array}{l}\text { Taxa de } \\
\text { mortalidade não } \\
\text { corrigida }\end{array}$ & $\begin{array}{c}\text { Número de } \\
\text { óbitos corrigido }\end{array}$ & $\begin{array}{c}\text { Taxa de } \\
\text { mortalidade } \\
\text { corrigida }\end{array}$ & \% correção \\
\hline Acre & 7 & 380 & 88,9 & 428 & 100,2 & 12,7 \\
\hline Alagoas & 40 & 5.403 & 312,1 & 5.891 & 340,2 & 9,0 \\
\hline Amapá & 4 & 653 & 144,7 & 807 & 178,7 & 23,5 \\
\hline Amazonas & 33 & 3.247 & 136,6 & 3.938 & 165,7 & 21,3 \\
\hline Bahia & 169 & 14.603 & 184,6 & 19.062 & 241,0 & 30,5 \\
\hline Ceará & 92 & 9.886 & 188,0 & 11.347 & 215,8 & 14,8 \\
\hline Distrito Federal & 1 & 2.214 & 118,4 & 2.223 & 118,9 & 0,4 \\
\hline Espírito Santo & 36 & 4.357 & 202,7 & 4.462 & 207,6 & 2,4 \\
\hline Goiás & 52 & 5.968 & 171,7 & 6.607 & 190,1 & 10,7 \\
\hline Maranhão & 90 & 4.521 & 119,5 & 5.983 & 158,2 & 32,3 \\
\hline Mato Grosso & 29 & 2.067 & 132,1 & 2.331 & 149,0 & 12,8 \\
\hline Mato Grosso do Sul & 25 & 1.048 & 78,9 & 1.085 & 81,6 & 3,5 \\
\hline Minas Gerais & 178 & 9.855 & 99,4 & 11.123 & 112,1 & 12,9 \\
\hline Pará & 101 & 8.361 & 154,4 & 10.308 & 190,4 & 23,3 \\
\hline Paraíba & 30 & 3.498 & 222,5 & 3.889 & 247,4 & 11,2 \\
\hline Paraná & 87 & 6.863 & 128,4 & 7.200 & 134,7 & 4,9 \\
\hline Pernambuco & 102 & 8.032 & 150,1 & 8.660 & 161,9 & 7,8 \\
\hline Piauí & 25 & 1.146 & 89,6 & 1.322 & 103,4 & 15,4 \\
\hline Rio de Janeiro & 65 & 11.385 & 117,5 & 12.659 & 130,6 & 11,2 \\
\hline Rio Grande do Norte & 27 & 2.988 & 189,1 & 3.667 & 232,1 & 22,7 \\
\hline Rio Grande do Sul & 100 & 4.788 & 88,2 & 5.136 & 94,6 & 7,3 \\
\hline Rondônia & 17 & 839 & 89,7 & 999 & 106,9 & 19,1 \\
\hline Roraima & 2 & 202 & 81,0 & 238 & 95,2 & 17,6 \\
\hline Santa Catarina & 61 & 1.498 & 45,3 & 1.655 & 50,0 & 10,5 \\
\hline São Paulo & 244 & 10.970 & 42,9 & 12.526 & 49,0 & 14,2 \\
\hline Sergipe & 24 & 1.873 & 163,1 & 2.048 & 178,3 & 9,3 \\
\hline Tocantins & 10 & 492 & 90,8 & 576 & 106,3 & 17,2 \\
\hline Brasil & 1,651 & 127.137 & 115,9 & 146.170 & 133,3 & 15,0 \\
\hline
\end{tabular}


No período estudado, a mediana da taxa de mortalidade corrigida de jovens por agressão nos municípios brasileiros com mais de 20 mil habitantes foi 71,5 por 100 mil habitantes. A Tabela 2 apresenta as taxas de mortalidade corrigidas, segundo características sociodemográficas dos municípios. Medianas das taxas superiores a 70 óbitos por 100 mil habitantes foram observadas nos municípios com mais de 50 mil habitantes, com até $90 \%$ de população residente em área urbana, com maior proporção de população de baixa renda ( $>7 \%$ ), com elevada desigualdade de renda (renda 20/40 acima de 12 vezes), com menor frequência de jovens no Ensino Médio (<45\%), com maior proporção de desocupação ( $>12 \%$ da população de 18-24 anos de idade desocupada) e com superioridade da população feminina em relação à masculina (razões de sexo masculino/feminino $<1$ ).

A Figura 2 apresenta graficamente as taxas de mortalidade corrigidas segundo porte populacional do município. As medianas das taxas de mortalidade são maiores à medida que aumenta o porte do município. As metrópoles apresentam a maior mediana da taxa de mortalidade (161,5 por 100 mil habitantes). O grupo dos municípios estudados de menor porte populacional (20.001 a 50 mil habitantes) apresentou a maior dispersão das taxas.

Tabela 2

Taxa de mortalidade corrigida por agressão de jovens de 15-29 anos de idade (por 100 mil habitantes), segundo características sociodemográficas dos municípios brasileiros com mais de 20 mil habitantes, 2010-2014.

\begin{tabular}{|c|c|c|c|c|c|}
\hline & $\begin{array}{l}\text { Número de } \\
\text { municípios }\end{array}$ & Média & Mediana & Q1 & Q3 \\
\hline \multicolumn{6}{|l|}{ Porte populacional (habitantes) } \\
\hline Pequeno porte 2 (20.001 a 50.000) & 1.043 & 83,8 & 61,7 & 30,8 & 109,2 \\
\hline Médio porte (50.001 a 100.000) & 325 & 108,4 & 87,7 & 40,3 & 155,0 \\
\hline Grande porte (100.001 a 900.000) & 266 & 139,1 & 102,7 & 53,4 & 204,0 \\
\hline Metrópoles (mais de 900.000) & 17 & 200,8 & 161,5 & 118,3 & 308,2 \\
\hline \multicolumn{6}{|c|}{ Proporção da população residente em área urbana } \\
\hline$\leq 65 \%$ & 489 & 84,9 & 70,0 & 38,9 & 113,4 \\
\hline $65 \%-90 \%$ & 604 & 103,7 & 76,9 & 36,3 & 146,4 \\
\hline$\geq 90 \%$ & 558 & 105,5 & 68,7 & 31,2 & 154,1 \\
\hline \multicolumn{6}{|l|}{ Proporção da população de baixa renda } \\
\hline$\leq 7 \%$ & 520 & 60,4 & 40,9 & 22,1 & 80,0 \\
\hline $7 \%-30 \%$ & 559 & 130,1 & 98,6 & 46,9 & 188,0 \\
\hline$\geq 30 \%$ & 572 & 103,0 & 83,0 & 45,8 & 134,2 \\
\hline \multicolumn{6}{|l|}{ Renda 20/40 } \\
\hline$\leq 9$ & 679 & 85,7 & 53,3 & 25,4 & 115,4 \\
\hline $9-12$ & 546 & 112,1 & 89,6 & 46,0 & 152,3 \\
\hline$\geq 12$ & 426 & 102,4 & 77,3 & 43,2 & 125,2 \\
\hline \multicolumn{6}{|c|}{ Taxa de frequência líquida ao ensino médio (15-17 anos) } \\
\hline$\geq 35 \%$ & 622 & 63,2 & 44,5 & 24,6 & 82,7 \\
\hline $35 \%-45 \%$ & 495 & 117,7 & 91,1 & 45,7 & 168,2 \\
\hline$\leq 35 \%$ & 534 & 122,6 & 96,1 & 52,5 & 158,7 \\
\hline \multicolumn{6}{|l|}{ Taxa de desocupação (18-24 anos) } \\
\hline$\leq 12 \%$ & 592 & 72,1 & 55,3 & 27,2 & 98,8 \\
\hline $12 \%-16 \%$ & 454 & 84,9 & 61,1 & 31,5 & 114,8 \\
\hline$\geq 16 \%$ & 605 & 135,2 & 101,4 & 55,4 & 184,8 \\
\hline \multicolumn{6}{|l|}{ Razão de sexos (masculino/feminino) } \\
\hline$>1,1$ & 57 & 76,5 & 56,6 & 30,4 & 117,9 \\
\hline $1,0-1,1$ & 526 & 85,4 & 63,0 & 33,6 & 111,5 \\
\hline$<1,0$ & 1.068 & 106,5 & 77,8 & 36,0 & 148,2 \\
\hline
\end{tabular}

Q1: primeiro quartil; Q3: terceiro quartil. 
Figura 2

Distribuição das taxas corrigidas de mortalidade por agressão de jovens de 15-29 anos de idade (por 100 mil habitantes) segundo porte populacional. Municípios brasileiros com mais de 20 mil habitantes, 2010-2014.

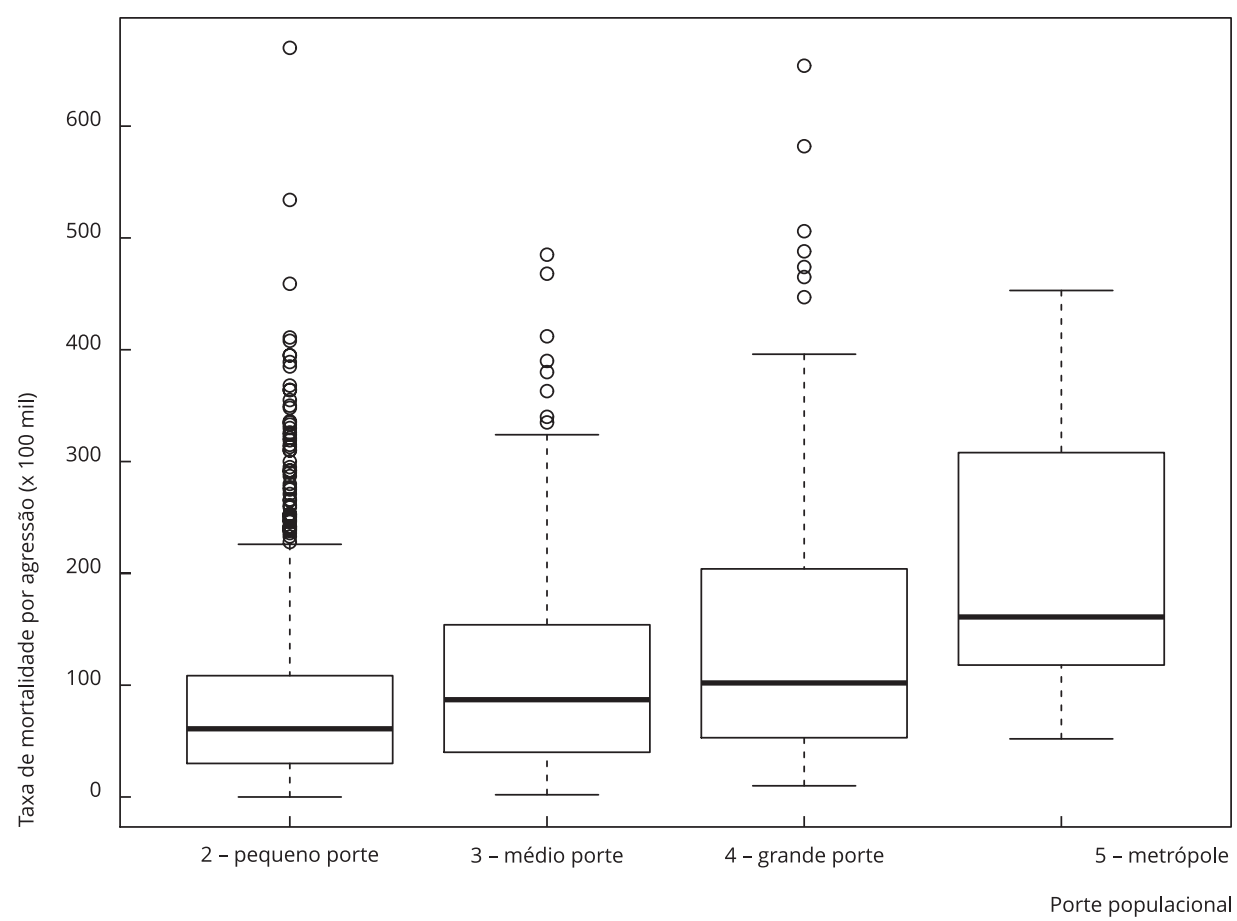

Quando examinadas as associações ecológicas entre as taxas de mortalidade corrigidas por agressão a jovens de 15-29 anos de idade do sexo masculino e as características sociodemográficas dos municípios brasileiros, no período de 2010-2014, observam-se alguns gradientes (Tabela 3). Na análise bruta, o risco de morte foi maior na medida em que aumentava o porte populacional do município, a proporção da população urbana, a proporção de jovens desocupados e a proporção da população feminina. Também foi maior quanto menor a frequência de jovens no Ensino Médio (Tabela 3).

$\mathrm{Na}$ análise ajustada (Tabela 3), houve associação direta entre mortalidade e urbanização (RTM = 1,37; IC95\%: 1,23-1,52 para municípios com proporção de população urbana 65 a 90\%, e RTM = 1,95; IC95\%: 1,70-2,23 para $\geq 90 \%$ ). Do mesmo modo, houve maior mortalidade nos municípios com as maiores proporções de população de baixa renda. Naqueles municípios onde a renda dos 20\% mais ricos superava entre 9 e 12 vezes a renda dos $40 \%$ mais pobres foram observadas taxas mais elevadas (RTM = 1,10; IC95\%: 1,01-1,20). Embora tenha havido associação entre renda 20/40 e mortalidade, não foi observada diferença entre as categorias de maior $(\geq 12)$ e menor $(\leq 9)$ desigualdade.

Evidenciou-se relação inversa entre mortalidade e proporção de jovens frequentando o ensino médio. O excesso de mortalidade foi maior nos municípios com $\leq 35 \%$ dos jovens de 18-24 anos frequentando o ensino médio (RTM = 2,05; IC95\%: 1,83-2,30). Observou-se um gradiente na associação entre desocupação e mortalidade, com riscos maiores nos municípios nos quais $\geq 16 \%$ dos jovens de 18-24 anos estavam desocupados (RTM = 1,27; IC95\%: 1,16-1,40). Municípios com maior número de mulheres em relação ao de homens (razão de sexos masculino/feminino < 1) registraram maior mortalidade de jovens do sexo masculino por agressões (RTM = 1,28; IC95\%: 1,05-1,58) (Tabela 3). 


\section{Tabela 3}

Associações ecológicas, brutas e ajustadas, entre as taxas de mortalidade corrigidas por agressão a jovens de $15-29$ anos de idade (por 100 mil habitantes) e características sociodemográficas dos municípios brasileiros com mais de 20 mil habitantes, $2010-2014$.

\begin{tabular}{|c|c|c|c|c|c|c|}
\hline \multirow[t]{2}{*}{ Variável } & \multicolumn{3}{|c|}{ Análise bruta } & \multicolumn{3}{|c|}{ Análise ajustada * } \\
\hline & RTM & $\mathrm{IC} 95 \%$ & Valor de p & RTM & $\mathrm{IC} 95 \%$ & Valor de p \\
\hline Porte populacional (habitantes) & & & $<0,001$ & - & - & - \\
\hline Pequeno porte 2 (20.001 a 50.000) & 1,00 & & & - & - & - \\
\hline Médio porte (50.001 a 100.000) & 1,29 & $1,17-1,43$ & & - & - & - \\
\hline Grande porte (100.001 a 900.000) & 1,66 & $1,49-1,85$ & & - & - & - \\
\hline Metrópoles (mais de 900.000) & 2,39 & $1,63-3,51$ & & - & - & - \\
\hline Proporção da população residente em área urbana & & & $<0,001$ & & & $<0,001$ \\
\hline$\leq 65 \%$ & 1,00 & & & 1,00 & & \\
\hline $65 \%-90 \%$ & 1,23 & $1,11-1,36$ & & 1,37 & $1,23-1,52$ & \\
\hline$\geq 90 \%$ & 1,25 & $1,13-1,38$ & & 1,95 & $1,70-2,23$ & \\
\hline Proporção da população de baixa renda & & & $<0,001$ & & & $<0,001$ \\
\hline$\leq 7 \%$ & 1,00 & & & 1,00 & & \\
\hline $7 \%-30 \%$ & 2,14 & $1,95-2,35$ & & 1,69 & $1,51-1,89$ & \\
\hline$\geq 30 \%$ & 1,68 & $1,53-1,85$ & & 1,52 & $1,29-1,78$ & \\
\hline Renda $20 / 40$ & & & $<0,001$ & & & 0,046 \\
\hline$\leq 9$ & 1,00 & & & 1,00 & & \\
\hline $9-12$ & 1,30 & $1,19-1,43$ & & 1,10 & $1,01-1,20$ & \\
\hline$\geq 12$ & 1,19 & $1,08-1,32$ & & 1,00 & $0,90-1,11$ & \\
\hline Taxa de frequência líquida ao ensino médio (15-17 anos) & & & $<0,001$ & & & $<0,001$ \\
\hline$\geq 45 \%$ & 1,00 & & & 1,00 & & \\
\hline $35 \%-45 \%$ & 1,85 & $1,69-2,03$ & & 1,67 & $1,52-1,84$ & \\
\hline$\leq 35 \%$ & 1,92 & $1,75-2,11$ & & 2,05 & $1,83-2,30$ & \\
\hline Taxa de desocupação (18 a 24 anos) & & & $<0,001$ & & & $<0,001$ \\
\hline$\leq 12 \%$ & 1,00 & & & 1,00 & & \\
\hline $12 \%-16 \%$ & 1,18 & $1,06-1,30$ & & 1,03 & $0,94-1,13$ & \\
\hline$\geq 16 \%$ & 1,87 & $1,71-2,05$ & & 1,27 & $1,16-1,40$ & \\
\hline Razão de sexos (masculino/feminino) & & & $<0,001$ & & & 0,001 \\
\hline$>1,1$ & 1,00 & & & 1,00 & & \\
\hline $1,0-1,1$ & 1,12 & $0,89-1,41$ & & 1,11 & $0,91-1,36$ & \\
\hline$<1,0$ & 1,40 & $1,11-1,75$ & & 1,28 & $1,05-1,58$ & \\
\hline
\end{tabular}

IC95\%: intervalos de 95\% de confiança; RTM: razões de taxas de mortalidade.

* Modelo final ajustado por regressão binomial negativa sem a inclusão da variável "porte populacional".

\section{Discussão}

Este trabalho revelou que, no período de 2010-2014, as taxas de mortalidade por agressão a jovens do sexo masculino foram elevadas no conjunto dos municípios brasileiros com mais de 20 mil habitantes. Ademais, taxas mais elevadas foram identificadas nos municípios maiores, mais urbanizados, com maiores proporções de pobreza, com menor proporção de jovens frequentando o ensino médio, com maior proporção de jovens de 18-24 anos desocupados e com maior número de mulheres em relação ao de homens.

As elevadas taxas de mortalidade por agressão corroboram achados prévios. No período de 20002012, no Brasil, foram registrados 958.224 óbitos de jovens de 15-29 anos de idade, 79,6\% do sexo masculino. Do total dos óbitos de homens jovens por causas externas ocorridos no período, 57\% foram devidos às agressões 4 . A importante magnitude e o incremento no risco de morte precoce por 
agressão, entre jovens do sexo masculino, nas últimas décadas, têm sido também objeto de estudo por diferentes autores 4,9,10,22. Nesse contexto, fatores como o desemprego, desestruturação familiar e a busca de padrões sociais, fruto das desigualdades, principalmente entre os jovens, contribuem para o aumento da violência 12,13,23.

Em comparação com outros países, a mortalidade de jovens por agressões é elevada. Na Região das Américas, em 2009, a taxa padronizada de mortalidade por agressões entre os homens, em todas as faixas etárias, foi 27,8/100 mil habitantes, sendo as faixas etárias de 15-24 e de 25-39 anos aquelas que contribuíram com o maior número de mortes 1 . No entanto, a exemplo dos Estados Unidos, desde 1993, as taxas de mortes por agressões entre os jovens de 15-19 anos de idade, ainda que elevadas, diminuíram significativamente, de 20,7 por 100 mil para 10,4 mortes por 100 mil em 200724 . Há grandes variações regionais nas taxas de mortalidade por violência entre os jovens. O Brasil, além de outros países da região da América Latina, Caribe e da África, apresenta taxas de mortalidade por violência entre os jovens pelo menos cem vezes mais altas do que aquelas em países da Europa Ocidental e do Pacífico Ocidental, onde se encontram as mais baixas taxas do mundo 25 .

Os achados do presente estudo foram consistentes com aqueles de outros trabalhos realizados no Brasil, quanto às maiores estimativas de risco de morte por agressão em municípios com maior urbanização 11,14,26,27. Segundo Duarte \& Barreto 28, o aumento da mortalidade por causas externas, observado a partir da década de 1980, deve-se aos homicídios em grandes centros urbanos, reforçando os pressupostos que as transições demográficas rápidas alimentam a violência.

É importante destacar que, além da urbanização, existem outros determinantes sociais da violência que possivelmente contribuem para explicar a mortalidade de jovens por agressões nos municípios, que não foram investigados no presente estudo. Por exemplo, um estudo sobre as condições sociais e o risco de homicídios em homens de 10-24 anos de idade, em municípios do Paraná e Santa Catarina (Brasil), verificou maior risco de mortes por homicídios entre os municípios de regiões de saúde que fazem fronteira com o Paraguai em relação aos municípios de outras áreas, sugerindo a relação entre a violência e questões como o tráfico de drogas, contrabando de armas e outros produtos, característicos da região de fronteira com o Paraguai 26. No Município de Belo Horizonte, Minas Gerais, Brasil, também foi observado risco mais elevado de morte por homicídios em determinadas regiões dominadas pelo tráfico de drogas e pelo crime organizado 29.

É consenso na literatura que a ocorrência de violência é maior nos espaços geográficos de importante desigualdade social. Wilkinson \& Pickett 30, em estudo abrangente, evidenciaram que quase todos os desfechos, incluindo a violência, são determinados não somente pela riqueza de uma sociedade, mas a partir de um determinado nível de riqueza, pela magnitude das desigualdades. Discutiram, ainda, que a violência não é relacionada apenas à criminalidade, mas a todos os problemas mais comuns nas camadas inferiores da sociedade, como gravidez na adolescência, uso de drogas, doenças mentais, mau desempenho na educação, prisão. Neste estudo, maiores estimativas de risco de morte por agressão foram identificadas nos municípios com maior pobreza e na categoria intermediária de desigualdade de renda. Esse achado pode ser atribuído ao fato de que os municípios mais pobres e mais desiguais são os de menores portes populacionais, os mesmos que apresentaram as taxas de mortalidade de homens jovens por agressões mais baixas no período do presente estudo, cujo modelo final não foi ajustado para a variável porte do município.

A literatura aponta resultados distintos quanto aos indicadores de renda e desigualdade associados à mortalidade por agressão nos municípios. Um estudo conduzido com os homens jovens dos municípios do Paraná, no período de 2002-2004, identificou correlação entre mortalidade por homicídios e o índice de Gini, e ausência de correlação com proporção de pobreza 31. Um estudo ecológico, com homens de 20-39 anos de idade, apontou que nos municípios com grande desigualdade de renda o risco de morte apresentou excesso em relação àqueles onde este indicador de desigualdade era mais discreto 14. De forma semelhante, Resende \& Andrade 32, ao analisarem crimes registrados em boletins de ocorrência da Secretaria Nacional de Segurança Pública em municípios brasileiros com população superior a cem mil habitantes, verificaram que a desigualdade de renda foi associada aos homicídios, embora não tenha influenciado na ocorrência de outros crimes como estupro, lesão corporal e tentativa de homicídio.

Maiores estimativas de risco de morte por agressão foram identificadas nos municípios com menor proporção de jovens de 15-17 anos de idade frequentando o Ensino Médio. De forma seme- 
lhante, Sousa et al. 33, ao investigarem a mortalidade por agressão e os determinantes sociais nos bairros do Município de Fortaleza (Ceará), no período de 2004-2006, apontaram que a baixa escolaridade (aferida pela média de anos de estudos e percentual de chefes de família com 15 ou mais anos de estudos) apresentou forte associação com a mortalidade por agressões.

Cabe destacar que, no Brasil, a educação é direito constitucional, reforçado no Estatuto da Juventude 15. No período de 2004-2014, a frequência escolar entre os jovens de 15-17 anos aumentou de $81,8 \%$ para $84,3 \% 34$. O atraso escolar entre jovens dessa faixa etária tem impacto sobre outra medida - a taxa de conclusão do Ensino Médio - que é um dos indicadores propostos para monitorar os Objetivos do Desenvolvimento Sustentável (ODS) 35. O abandono do estudo e a inserção precoce no mercado de trabalho contribuem para o afastamento do jovem do processo de socialização. Esse resultado causa maior preocupação porque a escola é um dos locais onde intervenções para reduzir o problema das violências podem ser realizadas.

Neste trabalho, maiores estimativas de risco de morte foram identificadas nos municípios com maior proporção de jovens de 18-24 anos sem emprego. Apesar de não ser consenso na literatura o efeito do desemprego entre os jovens sobre os homicídios 36 , outros trabalhos corroboram a associação encontrada 13,37. Um estudo ecológico sobre a evolução dos homicídios e indicadores de segurança pública no Município de São Paulo, entre 1996 e 2008, observou que a associação entre homicídios e desemprego apresentou maior magnitude quando comparada às associações com os indicadores de segurança pública, e que a redução em um ponto porcentual na taxa de desemprego esteve associada a uma redução de cerca de $10 \%$ no número de óbitos 12 .

Este estudo revelou maiores taxas de mortalidade de jovens por agressões em municípios com menor proporção de jovens frequentando o ensino médio e maior proporção dos que estão desempregados. Embora este trabalho não tenha investigado essa associação em nível individual, reitera-se a importância da atenção à chamada geração "nem-nem”, definida pelo IBGE como um grupo de jovens que não frequentava escola de ensino regular e nem trabalhava na semana de referência. Em 2014, praticamente um a cada cinco jovens fazia parte desse grupo 34.

Os achados do presente estudo foram consistentes com aqueles que evidenciam as maiores estimativas de risco de morte por agressão em municípios com maior número de mulheres em relação ao de homens 14,26. Em 2014, a razão de sexo foi de 93,9\% para o Brasil, e todas as Regiões Metropolitanas apresentaram razão inferior a 100\%, ou seja, maior número de mulheres que de homens. Em todos os países do mundo nascem mais homens do que mulheres, no entanto, como a sobremortalidade dos homens é maior em todas as faixas etárias, a proporção de homens tende a diminuir com o aumento da idade. Por esse motivo, uma população que possui uma maior idade média tende a ter uma menor razão de sexo 34 .

Destaca-se que este estudo apresenta limitações. Ressaltamos aquelas relacionadas ao uso de dados secundários do SIM, apesar da melhoria da qualidade e da ampliação da cobertura deste sistema, observadas a partir da década de 1990 37. Visando a corrigir a provável subnotificação da mortalidade por agressões, no presente trabalho, foi realizada correção mediante redistribuição proporcional dos eventos cuja intenção é indeterminada. A limitação relacionada à cobertura foi minimizada por meio do uso de fatores de correção do sub-registro propostos por Szwarcwald et al. 18. Não obstante, os fatores empregados foram estimados para mortalidade geral nas UF e utilizados, no presente estudo, para a correção da mortalidade específica de jovens do sexo masculino por agressões nos municípios, o que é outra limitação do estudo.

Este trabalho apresenta as limitações inerentes aos estudos ecológicos transversais, se limitando a apontar hipóteses explicativas para as mortes de jovens por agressão. Entre essas limitações, destacam-se as dificuldades de definição de temporalidade, por exemplo, avaliar se as maiores estimativas de risco de morte por agressão em municípios com maior número de mulheres em relação ao de homens (razão de sexo) são causa ou consequência das mortes por agressão, e de atribuição ao nível individual na maioria das associações observadas. A variável porte populacional, embora fortemente associada à mortalidade de jovens por agressões, não foi incluída no modelo devido à impossibilidade de se obter um modelo bem ajustado com esta variável. Ademais, a existência de colinearidade com a proporção da população residente em área urbana, tendo em vista que há uma associação inversamente proporcional entre área rural e o porte populacional do município, justifica a não inclusão de ambas as variáveis no modelo final. 
Outras limitações estão relacionadas às fontes de dados. Uma delas refere-se ao tamanho da população específica, cujas estimativas não estavam disponíveis. A aplicação da fração correspondente à população jovem do sexo masculino, estimada com base no Censo Demográfico 2010, a população total estimada para cada ano pelo IBGE para o TCU foi a alternativa possível. Acredita-se que não tenha introduzido vieses importantes aos resultados, tendo em vista que a presente análise se restringiu aos municípios com mais de 20 mil habitantes, que estão sujeitos a menos flutuações nos indicadores do que os municípios menores. Cabe destacar também a impossibilidade de levar em consideração o movimento migratório de homens jovens em busca de empregos em outras cidades, e seu retorno aos municípios de origem, no cenário da crise econômica no Brasil a partir de 2012. Nesse sentido, também deve-se mencionar que os dados sobre os óbitos são referentes ao quinquênio 2010-2014, já os indicadores socioeconômicos são derivados de dados do Censo Demográfico 2010. A ausência de indicadores anuais impossibilita captar mudanças no período do estudo, como os efeitos da crise econômica sobre os indicadores de renda e desigualdade nos municípios. Contudo, a validade dos resultados não é comprometida, tendo em vista que as taxas de mortalidade por agressões são influenciadas por processos sociohistóricos, não somente por exposições atuais.

A violência é um fenômeno multicausal que apresenta associação com fatores de risco individuais e familiares, mas também está relacionada fortemente com fatores sociais, culturais e ambientais 38,39 . Outros estudos ainda são necessários para que melhor se compreenda a multiplicidade de contextos e fatores associados a esse problema de saúde pública, e que considerem os possíveis impactos de crises econômicas, utilizando-se modelos multiníveis. Além disso, sugere-se que estudos desta natureza sejam realizados também com mulheres, entendendo-se que há diferenças de sexo importantes nos fatores associados à violência.

Em conclusão, a mortalidade de jovens do sexo masculino por agressão foi elevada, especialmente nos municípios maiores, mais urbanizados e com maior proporção de jovens buscando emprego e fora do ensino médio. Esses achados alertam para a necessidade de implementação de políticas voltadas a essa população específica. Em particular, evidencia-se a necessidade de garantir o acesso dos jovens ao ensino médio e estratégias para reduzir o abandono escolar. Investimentos para fornecer condições adequadas ao ensino, ampliar a cobertura do ensino em tempo integral, com práticas de atividades corporais, além de outras estratégias de ensino dinâmico e integrado, visando a apoiar o desenvolvimento global dos jovens, potencialmente resultariam em impacto de redução do risco de mortes prematuras por violência no país.

\section{Colaboradores}

A. C. M. Melo contribuiu na concepção e delineamento do estudo, na análise estatística, interpretação dos resultados e redação do manuscrito. G. D. M. Silva contribuiu na análise estatística, interpretação dos resultados e revisão do manuscrito. L. P. Garcia contribuiu na concepção e delineamento do estudo, interpretação dos resultados e revisão crítica do conteúdo do manuscrito.

\section{Agradecimentos}

À Coordenação Geral de Informações e Análises Epidemiológicas do Ministério da Saúde (CGIAE/ MS) pela disponibilização de dados utilizados na elaboração deste trabalho. 


\section{Referências}

1. Gawryszewski VP, Sanhueza A, Martinez-Piedra R, Escamilla JA, Souza MFM. Homicídios na região das Américas: magnitude, distribuição e tendências, 1999-2009. Ciênc Saúde Coletiva 2012; 17:3171-82.

2. Instituto Brasileiro de Geografia e Estatística. Síntese de indicadores sociais: uma análise das condições de vida da população brasileira: 2014. Rio de Janeiro: Instituto Brasileiro de Geografia e Estatística; 2014.

3. Pan American Health Organization. Leading cause of death. http://www.paho.org/data/in dex.php/en/indicators-mortality/mnu-lcd-en. html (acessado em 01/Set/2016).

4. Neves ACM, Garcia LP. Mortalidade de jovens brasileiros: perfil e tendências no período 2000-2012. Epidemiol Serv Saúde 2015; 24:595-606.

5. Reichenheim ME, Souza ER, Moraes CL, Mello Jorge MHP, Silva CMFP, Souza Minayo MC. Violence and injuries in Brazil: the effect, progress made, and challenges ahead. Lancet 2011; 377:1962-75

6. Krug E, Mercy J, Dahlberg L, Zwi A. The world report on violence and health. Lancet 2002; 360:1083-8.

7. Minayo MCS. Violência: um problema para a saúde dos brasileiros. In: Ministério da Saúde, organizador. Impacto da violência na saúde dos brasileiros. Brasília: Ministério da Saúde; 2005. p. 9-41.

8. Moura EC, Gomes R, Falcão MTC, Schwarz E, Neves ACM, Santos W. Gender inequalities in external cause mortality in Brazil, 2010. Ciênc Saúde Coletiva 2015; 20:779-88.

9. Souza ER, Gomes R, Silva JG, Correia BSC, Silva MMA. Morbimortalidade de homens jovens brasileiros por agressão: expressão dos diferenciais de gênero. Ciênc Saúde Coletiva 2012; 17:3243-8

10. Neves ACM, Garcia LP. Atendimentos de jovens vítimas de agressões em serviços públicos de urgência e emergência, 2011: diferenças entre sexos. Ciênc Saúde Coletiva 2017; 22: 1333-41.

11. Lima MLC, Ximenes RAA, Souza ER, Luna CF, Albuquerque MFPM. Análise espacial dos determinantes socioeconômicos dos homicídios no Estado de Pernambuco. Rev Saúde Pública 2005; 39:176-82.

12. Peres MFT, Almeida JF, Vicentin D, Ruotti C, Nery MB, Cerda M, et al. Evolução dos homicídios e indicadores de segurança pública no Município de São Paulo entre 1996 e 2008: um estudo ecológico de séries temporais. Ciênc Saúde Coletiva 2012; 17:3249-57.

13. Bando DH, Lester D. An ecological study on suicide and homicide in Brazil. Ciênc Saúde Coletiva 2014; 19:1179-89.

14. Duarte EC, Garcia LP, Freitas LRS, Mansano NH, Monteiro RA, Ramalho WM. Associação ecológica entre características dos municípios e o risco de homicídios em homens adultos de 20-39 anos de idade no Brasil, 1999-2010. Ciênc Saúde Coletiva 2012; 17:2259-68.
15. Brasil. Lei no 12.852 , de 5 de agosto de 2013. Institui o Estatuto da Juventude e dispõe sobre os direitos dos jovens, os princípios e diretrizes das políticas públicas de juventude e o Sistema Nacional de Juventude - SINAJUVE. Diário Oficial da República Federativa do Brasil 2013; 6 ago.

16. Melo DLB, Cano I. Índice de homicídios na adolescência: IHA 2012. Rio de Janeiro: Observatório de Favelas; 2014.

17. Cano I, Santos N. Violência letal, renda e desigualdade social no Brasil. Rio de Janeiro: 7 Letras; 2001.

18. Szwarcwald CL, Morais Neto OL, Escalante JJC, Souza Júnior PRB, Frias PG, Lima RB, et al. Busca ativa de óbitos e nascimentos no Nordeste e na Amazônia Legal: estimação das coberturas do SIM e do SINASC nos municípios brasileiros. In: Ministério da Saúde, organizador. Saúde Brasil 2010: uma análise da situação de saúde e de evidências selecionadas de impacto de ações de vigilância em saúde. Brasília: Ministério da Saúde; 2011. p. 79-98. (Série G. Estatística e Informação em Saúde).

19. Departamento de Vigilância de Doenças e Agravos Não Transmissíveis e Promoção da Saúde, Secretaria de Vigilância em Saúde, Ministério da Saúde. Saúde Brasil 2014: uma análise da situação de saúde e das causas externas. Brasília: Ministério da Saúde; 2015.

20. Paula GA. Modelos de regressão com apoio computacional. São Paulo: Universidade de São Paulo; 2013.

21. Montgomery DC. Design and analisys of experiments. $8^{\text {th }}$ Ed. New York; John Wiley \& Sons; 2013

22. Vermelho LL, Mello Jorge MHP. Mortalidade de jovens: análise do período de 1930 a 1991 (a transição epidemiológica para a violência). Rev Saúde Pública 1996; 30:319-31.

23. Souza ER, Lima MLC. Panorama da violência urbana no Brasil e suas capitais. Ciênc Saúde Coletiva 2006; 11:1211-22.

24. Blum RW, Qureshi F. Morbidity and mortality among adolescents and young adults in the United States. Baltimore: John Hopkins Bloomberg School of Public Health; 2011. (AstraZeneca Fact Sheet, 2011).

25. World Health Organization. Preventing youth violence: an overview of the evidence. Geneva: World Health Organization; 2015.

26. Mansano NH, Gutierrez MMU, Ramalho W, Duarte EC. Homicídios em homens jovens de 10 a 24 anos e condições sociais em municípios do Paraná e Santa Catarina, Brasil, 20012010. Epidemiol Serv Saúde 2013; 22:203-14.

27. Sumner SA, Mercy JA, Dahlberg LL, Hillis SD, Klevens J, Houry D. Violence in the United States. JAMA 2015; 314:478.

28. Duarte EC, Barreto SM. Transição demográfica e epidemiológica: a epidemiologia e serviços de saúde revisita e atualiza o tema. Epidemiol Serv Saúde 2012; 21:529-32. 
29. Beato Filho CC, Assunção RM, Silva BFA, Marinho FC, Reis IA, Almeida MCM. Conglomerados de homicídios e o tráfico de drogas em Belo Horizonte, Minas Gerais, Brasil, de 1995 a 1999. Cad Saúde Pública 2001; 17:1163-71.

30. Wilkinson R, Pickett K. O Nível: por que uma sociedade mais igualitária é melhor para todos. Rio de Janeiro: Editora Civilização Brasileira; 2015.

31. Andrade SM, Soares DA, Souza RKT, Matsuo T, Souza HD. Homicídios de homens de 15 a 29 anos e fatores relacionados no Estado do Paraná, de 2002 a 2004. Ciênc Saúde Coletiva 2011; 16:1281-8.

32. Resende JP, Andrade MV. Crime social, castigo social: desigualdade de renda e taxas de criminalidade nos grandes municípios brasileiros. Estudos Econômicos (São Paulo) 2011; 41:173-95.

33. Sousa GS, Magalhães FB, Gama IS, Lima MVN, Almeida RLF, Vieira LJES, et al. Social determinants and their interference in homicide rates in a city in northeastern Brazil. Rev Bras Epidemiol 2014; 17:194-203.

34. Instituto Brasileiro de Geografia e Estatística. Síntese de indicadores sociais : uma análise das condições de vida da população brasileira: 2015. Rio de Janeiro: Instituto Brasileiro de Geografia e Estatística; 2015.
35. Organização das Nações Unidas. 17 Objetivos de Desenvolvimento Sustentável. http:// nacoesunidas.org/pos2015/ (acessado em 01/ Set/2016).

36. Levitt SD. Understanding why crime fell in the 1990s: four factors that explain the decline and six that do not. J Econ Perspect 2004; 18: 163-90.

37. Garcia LP, Montenegro MMS, Ramalho WM. Mortalidade no Brasil: situação de 2008 e evolução segundo principais grupos de causas no período de 1980 a 2008. In: Ministério da Saúde, organizador. Saúde Brasil 2009: uma análise da situação de saúde e da agenda nacional e internacional de prioridades em saúde. Brasília: Ministério da Saúde; 2010. p. 45-72. (Série G. Estatística e Informação em Saúde).

38. Kann L, Kinchen S, Shanklin SL, Flint KH, Kawkins J, Harris WA, et al. Youth risk behavior surveillance--United States, 2013. MMWR Suppl 2014; 63:1-168.

39. Dahlberg LL, Krug EG. Violência: um problema global de saúde pública. Ciênc Saúde Coletiva 2006; 11:1163-78. 


\section{Abstract}

The aim was to analyze the association between risk of death from assault in young males and socio-demographic characteristics in Brazilian municipalities. In this ecological study, the units of analysis were the 1,651 municipalities of Brazil with more than 20,000 inhabitants. Data were obtained from the Brazilian Mortality Information System (SIM) and indicators were obtained from the 2010 Population Census and Human Development Atlas. Mortality rate ratios were estimated by a negative binomial regression model. From 2010 to 2014, a total of 127,137 deaths from assault were reported in young males 15 to 29 years of age. Corrected mortality rate was 133.3/100 thousand inhabitants for the set of municipalities (median 71.5/100 thousand inhabitants). The rate increased with the municipalities' population size. In the adjusted model, higher rates ratios were observed in the more urbanized municipalities (1.95; 95\% CI: 1.70-2.23), in intermediate categories of income inequality (1.10; 95\% CI: 1.01-1.20) and poverty rate (1.69; 95\%CI: 1.51-1.89), with lower proportion of youth attending Secondary School (2.05; 95\%CI: 1.83-2.30), with higher proportion of unemployed youth 18 to 24 years of age (1.27; 95\% CI: 1.16-1.40), and with more women than men (1.28; 95\%CI: 1.05-1.58). Mortality from assault was high in young Brazilian men, especially in larger and more urbanized municipalities and those with a higher proportion of youth looking for work and not attending secondary school. The results show the relevance of social policies for dealing with violence against youth.

Homicide; Violence; Health Inequalities; Men

\section{Resumen}

El objetivo fue analizar la asociación entre el riesgo de muerte por agresiones en jóvenes del sexo masculino y características sociodemográficas de los municipios brasileños. Estudio ecológico teniendo como unidades de análisis los 1.651 municipios con más de 20.000 habitantes. Se utilizaron datos del Sistema de Información sobre Mortalidad (SIM) e indicadores obtenidos del Censo Demográfico 2010 y del Atlas de Desarrollo Humano. Las razones de tasas de mortalidad se estimaron por el modelo de regresión binomial negativa. En el período de 2010-2014, se registraron 127.137 óbitos por agresión a jóvenes de 15-29 años de edad del sexo masculino. La tasa corregida de mortalidad fue de 133,3/100 mil habitantes en el conjunto de los municipios (mediana 71,5/100 mil habitantes). La tasa fue mayor, a medida que aumentó el porte poblacional de los municipios. Razones de tasas más elevadas en el modelo ajustado se observaron en los municipios más urbanizados (1,95; IC95\%: 1,70-2,23), en categorías intermedias de desigualdad de renta (1,10; IC95\%: 1,01-1,20) y proporción de pobreza (1,69; IC95\%: 1,51-1,89), con menor proporción de jóvenes frecuentando la Enseñanza Media (2,05; IC95\%: 1,83-2,30), mayor proporción de jóvenes de 1824 años desocupados (1,27; IC95\%: 1,16-1,40) y mayor número de mujeres en relación a hombres (1,28; IC95\%: 1,05-1,58). La mortalidad de jóvenes del sexo masculino por agresión fue elevada, especialmente en los municipios mayores, más urbanizados y con mayor proporción de jóvenes buscando empleo y fuera de la enseñanza media. Se evidencia la relevancia de las políticas sociales para el enfrentamiento de la violencia entre jóvenes.

Homicidio; Violencia; Desigualdades en la Salud; Hombres
Recebido em 29/Set/2016

Versão final reapresentada em 06/Jan/2017

Aprovado em 01/Fev/2017 